\title{
Short sums of restricted Möbius functions
}

by

\section{Olivier Bordellès (Aiguilhe)}

1. Introduction and result. In what follows, $10 \leq y \leq x$ are large real numbers, $e(t)=e^{2 \pi i t}$, [t] is the integer part of $t$ and $\psi(t)=t-[t]-1 / 2$. Finally, $\varepsilon>0$ is an arbitrary small real number which does not need to be the same at each occurrence.

In 1976, Ramachandra [12] proved a general theorem for short sums of certain multiplicative functions from which he deduced that

$$
\sum_{x<n \leq x+y} \mu(n)=O\left(x^{1-1 / B+\varepsilon}+y \exp \left(-(\log x)^{1 / 6}\right)\right)
$$

where $\mu(n)$ is the Möbius function and $B \geq 2$ is an admissible absolute constant occurring in zero-density estimates. From the work of Huxley [8], we know that $B=12 / 5$ is admissible so that we have

$$
\sum_{x<n \leq x+y} \mu(n)=O\left(x^{7 / 12+\varepsilon}+y \exp \left(-(\log x)^{1 / 6}\right)\right) .
$$

The density hypothesis states that $B=2$ is admissible, so that

$$
\sum_{x<n \leq x+y} \mu(n)=O\left(x^{1 / 2+\varepsilon}+y \exp \left(-(\log x)^{1 / 6}\right)\right)
$$

if the density hypothesis is true.

It should be mentioned that (1) was also independently discovered by Motohashi [11, and that the paper of Ramachandra was later refined (see [13, 14]) and generalized to problems in number fields (see [5]).

From (1) we could easily infer that if $x^{7 / 12+\varepsilon} \leq y \leq x$ then

$$
\sum_{x<n \leq x+y} \mu(n)=o(y)
$$

2010 Mathematics Subject Classification: 11A25, 11L07.

Key words and phrases: short sums, restricted Möbius functions, exponential sums estimates. 
unconditionally. Using the important identity $\sum_{d \mid n} \mu(d)=0$ valid for any integer $n>1$, we can write

$$
\sum_{x<n \leq x+y} \mu(n)=-\sum_{x<n \leq x+y} M(n ; x)
$$

where we set

$$
M(n ; t):=\sum_{\substack{d \mid n \\ d \leq t}} \mu(d)
$$

so that (3) could be written as

$$
\sum_{x<n \leq x+y} M(n ; x)=o(y)
$$

for $x^{7 / 12+\varepsilon} \leq y \leq x$ unconditionally. With (2) and (4) in mind, this paper deals with the following slightly different version of this problem: we ask for the greatest exponent $\theta \in(0,1]$ so that the estimate

$$
\sum_{x<n \leq x+y} M\left(n ; x^{\theta}\right)=o(y)
$$

holds true for $x^{1 / 2+\varepsilon} \leq y \leq x$. If the density hypothesis is true, then $\theta=1$ is admissible. Unconditionally, the answer depends on estimates of twisted exponential sums of types I and II. This leads to the following result:

TheOREM 1.1. Let $x^{1 / 2+6 \varepsilon} \leq y \leq x$ be large real numbers. Then

$$
\sum_{x<n \leq x+y} M\left(n ; x^{4 / 7}\right)=y \sum_{d \leq x^{4 / 7}} \frac{\mu(d)}{d}+O_{\varepsilon}\left(y x^{-\varepsilon}\right) .
$$

\section{The sums $\sum_{n} \mu(n) \psi(x / n)$}

2.1. Introduction of exponential sums. In this section,

$$
\|t\|=\min \{1 / 2+\psi(t), 1 / 2-\psi(t)\}
$$

is the distance of $t$ to the nearest integer. We begin with the following result:

Proposition 2.1. Let $x$ be a sufficiently large real number, $\varepsilon>0$ be a small real number and $4 \leq H \leq R \leq x$ be integers. Then

$$
\sum_{R<n \leq 2 R} \mu(n) \psi\left(\frac{x}{n}\right)=-\sum_{0<|h| \leq H} \frac{1}{2 \pi i h} \sum_{R<n \leq 2 R} \mu(n) e\left(\frac{h x}{n}\right)+O_{\varepsilon}\left(R H^{-1} x^{\varepsilon}\right) .
$$

The proof needs the following two lemmata:

Lemma 2.2. Let $N \geq 1$ and $H \geq 4$ be integers, and $f:[N, 2 N] \rightarrow \mathbb{R}$ be any map. For any real number $0<\delta \leq 1 / 4$ set

$$
\mathcal{R}(f, N, \delta):=|\{n \in(N, 2 N] \cap \mathbb{Z}:\|f(n)\|<\delta\}|
$$


and let $K:=[\log H / \log 2]$. Then

$$
\sum_{N<n \leq 2 N} \min \left(1, \frac{1}{H\|f(n)\|}\right)<24 N H^{-1}+2 \sum_{k=0}^{K-2} 2^{-k} \mathcal{R}\left(f, N, 2^{k} H^{-1}\right) .
$$

Proof. We have

$$
\begin{aligned}
\sum_{N<n \leq 2 N} \min \left(1, \frac{1}{H\|f(n)\|}\right) & =\sum_{\substack{N<n \leq 2 N \\
\|f(n)\|<H^{-1}}} 1+\frac{1}{H} \sum_{\substack{N<n \leq 2 N \\
\|f(n)\| \geq H^{-1}}} \frac{1}{\|f(n)\|} \\
& =\mathcal{R}\left(f, N, H^{-1}\right)+\frac{1}{H} \sum_{\substack{N<n \leq 2 N \\
\|f(n)\| \geq H^{-1}}} \frac{1}{\|f(n)\|} .
\end{aligned}
$$

Since

$$
\begin{aligned}
\{n \in(N, 2 N] & \left.\cap \mathbb{Z}:\|f(n)\| \geq H^{-1}\right\} \\
& \subseteq \bigcup_{k=1}^{K}\left\{n \in(N, 2 N] \cap \mathbb{Z}: 2^{k-1} H^{-1} \leq\|f(n)\|<2^{k} H^{-1}\right\}
\end{aligned}
$$

we get

$$
\begin{aligned}
& \sum_{\substack{N<n \leq 2 N \\
\|f(n)\| \geq H^{-1}}} \frac{1}{\|f(n)\|} \leq \sum_{k=1}^{K} \sum_{\substack{N<n \leq 2 N \\
2^{k-1} H^{-1} \leq\|f(n)\|<2^{k} H^{-1}}} \frac{1}{\|f(n)\|} \\
& \quad \leq(N+1)\left(2^{1-K}+2^{2-K}\right) H+\sum_{k=1}^{K-2} \sum_{\substack{N<n \leq 2 N \\
2^{k-1} H^{-1} \leq\|f(n)\|<2^{k} H^{-1}}} \frac{1}{\|f(n)\|} \\
& \quad \leq 6 \cdot 2^{-K}(N+1) H+2 H \sum_{k=1}^{K-2} 2_{\substack{N<n \leq 2 N \\
\|f(n)\|<2^{k} H^{-1}}} 1 \\
& \quad<12(N+1)+2 H \sum_{k=1}^{K-2} 2^{-k} \mathcal{R}\left(f, N, 2^{k} H^{-1}\right)
\end{aligned}
$$

since $2^{-K}<2 H^{-1}$. Thus we get

$$
\begin{aligned}
\sum_{N<n \leq 2 N} \min \left(1, \frac{1}{H\|f(n)\|}\right)< & \mathcal{R}\left(f, N, H^{-1}\right)+24 N H^{-1} \\
& +2 \sum_{k=1}^{K-2} 2^{-k} \mathcal{R}\left(f, N, 2^{k} H^{-1}\right),
\end{aligned}
$$

which implies the desired result. 
LEMmA 2.3. Let $1 \leq y \leq x$ and $0<\varepsilon<1 / 2$ be real numbers. If $\tau(n)$ is the usual divisor function, then

$$
\sum_{x-y<n \leq x+y} \tau(n) \ll_{\varepsilon} y x^{\varepsilon} .
$$

Proof. If $1 \leq y \leq x^{\varepsilon}$ then

$$
\sum_{x-y<n \leq x+y} \tau(n) \leq(2 y+1) \max _{x-y<n \leq x+y} \tau(n) \ll_{\varepsilon} y x^{\varepsilon},
$$

and if $x^{\varepsilon}<y \leq x$ then the result is a consequence of Shiu's theorem [15].

Now we turn to the proof of Proposition 2.1.

Proof of Proposition 2.1. Since

$$
\psi(t)=-\sum_{0<|h| \leq H} \frac{e(h t)}{2 \pi i h}+O\left(\min \left(1, \frac{1}{H\|t\|}\right)\right)
$$

we easily see using Lemma 2.2 that

$$
\begin{aligned}
\sum_{R<n \leq 2 R} \mu(n) \psi\left(\frac{x}{n}\right)= & -\sum_{0<|h| \leq H} \frac{1}{2 \pi i h} \sum_{R<n \leq 2 R} \mu(n) e\left(\frac{h x}{n}\right) \\
& +O\left(\sum_{R<n \leq 2 R} \min \left(1, \frac{1}{H\|x / n\|}\right)\right) \\
= & -\sum_{0<|h| \leq H} \frac{1}{2 \pi i h} \sum_{R<n \leq 2 R} \mu(n) e\left(\frac{h x}{n}\right) \\
& +O\left(R H^{-1}+\sum_{k=0}^{[\log H / \log 2]-2} 2^{-k} \mathcal{R}\left(\frac{x}{n}, R, \frac{2^{k}}{H}\right)\right) .
\end{aligned}
$$

Now interchanging the summations and using Lemma 2.3 we obtain

$$
\begin{aligned}
\mathcal{R}\left(\frac{x}{n}, R, \frac{2^{k}}{H}\right) & \leq \sum_{R<n \leq 2 R}\left(\left[\frac{x}{n}+\frac{2^{k}}{H}\right]-\left[\frac{x}{n}-\frac{2^{k}}{H}\right]\right) \\
& \leq \sum_{x-2^{k+1} R H^{-1}<m \leq x+2^{k+1} R H^{-1}} \sum_{\substack{d \mid m \\
R<d \leq 2 R}} 1 \\
& \leq \sum_{x-2^{k+1} R H^{-1}<m \leq x+2^{k+1} R H^{-1}} \tau(m) \ll_{\varepsilon} 2^{k} R H^{-1} x^{\varepsilon},
\end{aligned}
$$

which proves Proposition 2.1 .

The following result improves slightly on Lemma 8 of [1]. 
COROLlary 2.4. Under the hypothesis of Proposition 2.1 with $10 \leq y$ $\leq x$ we have

$$
\begin{aligned}
& \sum_{R<n \leq 2 R} \mu(n)\left(\psi\left(\frac{x+y}{n}\right)-\psi\left(\frac{x}{n}\right)\right) \\
& \ll \frac{y}{R} \max _{R \leq R^{\prime} \leq 2 R} \max _{x \leq z \leq x+y} \max _{H_{1} \leq H}\left|\sum_{R<n \leq R^{\prime}} \mu(n) \sum_{H_{1}<h \leq 2 H_{1}} e\left(\frac{h z}{n}\right)\right| \log H \\
& \quad+R H^{-1} x^{\varepsilon} .
\end{aligned}
$$

Proof. Using Proposition 2.1 we get

$$
\begin{aligned}
\sum_{R<n \leq 2 R} \mu(n) & \left(\psi\left(\frac{x+y}{n}\right)-\psi\left(\frac{x}{n}\right)\right) \\
= & -\sum_{0<|h| \leq H} \frac{1}{2 \pi i h} \sum_{R<n \leq 2 R} \mu(n)\left\{e\left(\frac{h(x+y)}{n}\right)-e\left(\frac{h x}{n}\right)\right\} \\
& +O_{\varepsilon}\left(R H^{-1} x^{\varepsilon}\right),
\end{aligned}
$$

and the identity

$$
e(a(x+y))-e(a x)=2 \pi i a \int_{x}^{x+y} e(a t) d t
$$

and Abel summation give the asserted result.

2.2. Sums of types I and II. Corollary 2.4 reduces the problem to finding bounds for sums

$$
\sum_{n \sim R} \mu(n) \sum_{h \sim H} e\left(\frac{h x}{n}\right) .
$$

Such bounds are achieved by using clever identities discovered by Vaughan (see [10] for example) and generalized by Heath-Brown [6]. We sum up the process in Lemma 2.5 below (see also Lemma 2 of [3]). We consider integers $M, N, R, R^{\prime} \geq 1$ such that $R<R^{\prime} \leq 2 R$ and let $S>0$ be any real number. If $f:\left(R, R^{\prime}\right] \rightarrow \mathbb{C}$ is any function, it is convenient to define sums of type $I$ (related to $f$ ) to be the sums

$$
\mathcal{S}_{I}:=\sum_{\substack{M<m \leq 2 M \\ R<m n \leq R^{\prime}}} \sum_{\substack{N<n \leq 2 N \\ m}} a_{m} f(m n)
$$

and sums of type II (related to $f$ ) to be the sums

$$
\mathcal{S}_{I I}:=\sum_{\substack{M<m \leq 2 M \\ R<m n \leq R^{\prime}}} \sum_{\substack{N<n \leq 2 N\\}} a_{m} b_{n} f(m n)
$$


where $a_{m}, b_{n}$ are complex numbers supported respectively on $(M, 2 M]$ and $(N, 2 N]$ and satisfying $a_{m} \ll_{\varepsilon} m^{\varepsilon}$ and $b_{n} \ll_{\varepsilon} n^{\varepsilon}$.

Lemma 2.5. Suppose that the estimates

$$
\begin{aligned}
\mathcal{S}_{I} \ll S & \text { for } N \gg R^{1 / 2}, \\
\mathcal{S}_{I I} \ll S & \text { for } R^{1 / 3} \ll N \ll R^{1 / 2}
\end{aligned}
$$

hold true for all sums of type I and type II. Then

$$
\sum_{R<n \leq R^{\prime}} \mu(n) f(n) \ll S(\log 3 R)^{5} .
$$

It is well-known that the multiplicative restrictions $R<m n \leq R^{\prime}$ could be removed from sums $\mathcal{S}_{I}$ and $\mathcal{S}_{I I}$ at a cost of a factor $\log R$ (see [1, Lemma 15] for instance).

To treat sums of type I we appeal to the following result which is the estimate (5.9) of Corollary 8 from [9].

Lemma 2.6. Let $X>0$ be a real number, $H, M, N \geq 1$ be integers and $\alpha, \beta \in \mathbb{R}$ such that $\beta \neq-1,0$ and $\alpha /(1+\beta) \neq 0,1$. Let $I \subseteq(N, 2 N]$ and let $\left(a_{m}\right),\left(c_{h}\right) \in \mathbb{C}$ satisfy $\left|a_{m}\right|,\left|c_{h}\right| \leq 1$. Then for all $\varepsilon>0$,

$$
\begin{aligned}
& \sum_{H \leq h<2 H} \sum_{M \leq m<2 M} \sum_{n \in I} a_{m} c_{h} e\left(X\left(\frac{m}{M}\right)^{\alpha}\left(\frac{h N}{n H}\right)^{\beta}\right) \\
& \ll\left\{\left(X^{3} H^{6} M^{6} N^{2}\right)^{1 / 8}+H(X M)^{1 / 2}+H M\right. \\
&\left.+\left(X H^{3} N\right)^{1 / 4} M+X^{-1} H M N\right\}(H M N)^{\varepsilon} .
\end{aligned}
$$

In the last two decades, many authors provided nontrivial bounds for sums of type II. Among these we pick up the following estimate with the exponent pair $(k, l)=(1 / 2,1 / 2)$ (4], see also [2]). The idea of the proof goes back to Heath-Brown [7].

Lemma 2.7. Let $z>0$ be a real number, $H, M, N \geq 1$ be integers and let $\left(a_{m}\right),\left(B_{h, n}\right) \in \mathbb{C}$ satisfy $\left|a_{m}\right|,\left|B_{h, n}\right| \leq 1$. Set $L:=\log (2 H M N)$. Then

$$
\begin{aligned}
\sum_{M \leq m<2 M} \sum_{H \leq h<2 H} \sum_{N \leq n<2 N} a_{m} B_{h, n} e\left(\frac{h z}{m n}\right) \\
\ll\left\{H\left(z M^{3} N^{4}\right)^{1 / 6}+M(H N)^{1 / 2}\right. \\
\left.+M^{1 / 2} H N+\left(H z^{-1}\right)^{1 / 2}(M N)^{3 / 2}\right\} L^{3} .
\end{aligned}
$$

\section{Proof of Theorem 1.1}

Proposition 3.1. Let $x^{2 / 5} \leq y \leq x$ be real numbers, and $10 \leq R \leq x$ be a large integer. Then for every $\varepsilon>0$ we have 


$$
\begin{aligned}
& \sum_{R<n \leq 2 R} \mu(n)\left(\psi\left(\frac{x+y}{n}\right)-\psi\left(\frac{x}{n}\right)\right) \\
& \ll\left\{x^{1 / 12} y^{1 / 2} R^{7 / 24}+x^{-1 / 24} y^{3 / 4} R^{3 / 16}+x^{-1 / 12} y^{1 / 2} R^{11 / 24}+x^{-13 / 24} y^{3 / 4} R^{41 / 48}\right. \\
& \left.\quad+x^{1 / 32} y^{7 / 16} R^{-5 / 64}+x^{3 / 8} y^{1 / 4} R^{-3 / 16}+x^{-1} y R\right\} x^{\varepsilon} .
\end{aligned}
$$

Proof. Note that if $10 \leq R \leq\left(x^{2} y^{12}\right)^{1 / 17}$, then $x^{1 / 12} y^{1 / 2} R^{7 / 24} \geq R$ so that we may suppose $\left(x^{2} y^{12}\right)^{1 / 17}<R \leq x$. To treat the sum of Corollary 2.4. we apply Lemma 2.5 with

$$
f(n)=\sum_{H_{1}<h \leq 2 H_{1}} e\left(\frac{h z}{n}\right)
$$

where $R<n \leq R^{\prime}, 1 \leq H_{1} \leq H$ and $x \leq z \leq x+y$. Using Lemma 2.6 with $-\alpha=c_{h}=\beta=1, H=H_{1}, z=X M N H_{1}^{-1}$ and supposing that $M N \asymp R$ with $N \gg R^{1 / 2}$, we get

$\mathcal{S}_{I} \ll\left\{\left(z H_{1}^{9} R\right)^{1 / 8}+\left(z^{2} H_{1}^{6} R^{-1}\right)^{1 / 4}+H_{1} R^{1 / 2}+H_{1}\left(z^{2} R^{3}\right)^{1 / 8}+z^{-1} R^{2}\right\}\left(H_{1} R\right)^{\varepsilon}$

and, similarly, using Lemma 2.7 with $B_{h, n}=b_{n}, H=H_{1}$ and supposing that $M N \asymp R$ with $R^{1 / 3} \ll N \ll R^{1 / 2}$, we obtain

$\mathcal{S}_{I I} \ll\left\{H_{1}\left(z^{2} R^{7}\right)^{1 / 12}+H_{1}^{1 / 2} R^{5 / 6}+H_{1} R^{3 / 4}+\left(H_{1} z^{-1}\right)^{1 / 2} R^{3 / 2}\right\}\left(\log 2 H_{1} R\right)^{4}$

so that for every integer $4 \leq H \leq R$, we get, using Corollary 2.4 and Lemma 2.5.

$$
\begin{aligned}
& \sum_{R<n \leq 2 R} \mu(n)\left(\psi\left(\frac{x+y}{n}\right)-\psi\left(\frac{x}{n}\right)\right) \\
& \ll\left\{y H\left(x^{2} R^{-5}\right)^{1 / 12}+y H^{1 / 2} R^{-1 / 6}+y H R^{-1 / 4}+y\left(x^{-1} H^{9} R^{-7}\right)^{1 / 8}\right. \\
& \left.\quad+y\left(x^{2} H^{6} R^{-5}\right)^{1 / 4}+y H\left(x^{2} R^{-5}\right)^{1 / 8}+x^{-1} y R\right\}(H R)^{\varepsilon}+R H^{-1} x^{\varepsilon}
\end{aligned}
$$

Since $y \geq x^{2 / 5}$, we have $R>\left(x^{2} y^{12}\right)^{1 / 17} \geq x^{2 / 5}$, so that $y H\left(x^{2} R^{-5}\right)^{1 / 8}$ is dominated by the first term, and the choice of $H=\left[4 x^{-1 / 12} y^{-1 / 2} R^{17 / 24}\right]$ gives the desired result.

The following result is an easy consequence of Proposition 3.1 .

Corollary 3.2. If $x^{1 / 2+6 \varepsilon} \leq y \leq x$ then

$$
\max _{x^{1 / 2}<R \leq x^{4 / 7}} \sum_{R<n \leq 2 R} \mu(n)\left(\psi\left(\frac{x+y}{n}\right)-\psi\left(\frac{x}{n}\right)\right) \ll y x^{-2 \varepsilon} .
$$


Proof. Indeed, we get

$$
\begin{aligned}
& \max _{x^{1 / 2}<R \leq x^{4 / 7}} \sum_{R<n \leq 2 R} \mu(n)\left(\psi\left(\frac{x+y}{n}\right)-\psi\left(\frac{x}{n}\right)\right) \\
& \ll x^{1 / 4+\varepsilon} y^{1 / 2}+x^{11 / 168+\varepsilon} y^{3 / 4}+x^{9 / 32+\varepsilon} y^{1 / 4}+y x^{-3 / 7+\varepsilon} \ll y x^{-2 \varepsilon}
\end{aligned}
$$

since $x^{1 / 2+6 \varepsilon} \leq y \leq x$.

Now we are able to prove Theorem 1.1. Interchanging the summations we obtain

$$
\begin{aligned}
\sum_{x<n \leq x+y} M\left(n ; x^{4 / 7}\right) & =\sum_{d \leq x^{4 / 7}} \mu(d)\left(\left[\frac{x+y}{d}\right]-\left[\frac{x}{d}\right]\right) \\
= & y \sum_{d \leq x^{4 / 7}} \frac{\mu(d)}{d}-\sum_{d \leq x^{4 / 7}} \mu(d)\left(\psi\left(\frac{x+y}{d}\right)-\psi\left(\frac{x}{d}\right)\right) \\
= & y \sum_{d \leq x^{4 / 7}} \frac{\mu(d)}{d}-\sum_{x^{1 / 2}<d \leq x^{4 / 7}} \mu(d)\left(\psi\left(\frac{x+y}{d}\right)-\psi\left(\frac{x}{d}\right)\right) \\
& +O\left(x^{1 / 2}\right)
\end{aligned}
$$

and using Corollary 3.2 along with a splitting argument gives the result.

Acknowledgments. I express my gratitude to the referee for his careful reading of the manuscript.

\section{References}

[1] R. C. Baker, The greatest prime factor of the integers in an interval, Acta Arith. 47 (1986), 193-231.

[2] -, The square-free divisor problem, Quart. J. Math. Oxford 45 (1994), 269-277.

[3] - Sums of two relatively prime cubes, Acta Arith. 129 (2007), 103-146.

[4] X. Cao and W.-G. Zhai, The distribution of square-free numbers of the form $\left[n^{c}\right]$, J. Théorie Nombres Bordeaux 10 (1998), 287-299.

[5] M. D. Coleman, The Hooley-Huxley contour method for problems in number fields I: arithmetic functions, J. Number Theory 74 (1999), 250-277.

[6] D. R. Heath-Brown, Prime numbers in short intervals and a generalized Vaughan identity, Canad. J. Math. 34 (1982), 1365-1377.

[7] —, The Pjateckir-Šapiro prime number theorem, J. Number Theory 16 (1983), 242266.

[8] M. N. Huxley, On the difference between consecutive primes, Invent. Math. 15 (1972), 164-170.

[9] E. Kowalski, O. Robert and J. Wu, Small gaps in coefficients of L-functions and $\mathfrak{B}$-free numbers in short intervals, Rev. Mat. Iberoamer. 23 (2007), 281-326.

[10] H. L. Montgomery and R. C. Vaughan, The distribution of squarefree numbers, in: Recent Progress in Analytic Number Theory (Durham, 1979), Vol. 1, Academic Press, 1981, 247-256. 
[11] Y. Motohashi, On the sum of the Möbius function in a short segment, Proc. Japan Acad. 52 (1976), 477-479.

[12] K. Ramachandra, Some problems of analytic number theory I, Acta Arith. 31 (1976), 313-323.

[13] K. Ramachandra, A. Sankaranarayanan and K. Srinivas, Addendum to Ramachandra's paper "Some problems of analytic number theory I", ibid. 73 (1995), 367-371.

[14] A. Sankaranarayanan and K. Srinivas, On the papers of Ramachandra and Katai, ibid. 62 (1992), 373-382.

[15] P. Shiu, A Brun-Titchmarsh theorem for multiplicative functions, J. Reine Angew. Math. 313 (1980), 161-170.

Olivier Bordellès

2 allée de la Combe

43000 Aiguilhe, France

E-mail: borde43@wanadoo.fr 